\title{
La alfabetización universitaria y el contacto con las fuentes de información, claves para el aprendizaje en la universidad' ${ }^{1}$
}

\author{
University Literacy and Contact with Sources of Information, Keys for Learning \\ at The University ${ }^{2}$
}

\section{Alfabetização universitária e contato com as fontes de informação: chave para a formação acadêmica ${ }^{3}$}

\author{
Fabiana Böhm-Carrer ${ }^{4}$ \\ Universidad del Aconcagua \\ Mendoza, Argentina \\ bohmcarrer@yahoo.com \\ Adrián Edgardo Lucero 5 \\ Universidad Nacional det Cuyo \\ Mendoza, Argentina \\ adrian_lucero@inv.gov.ar
}

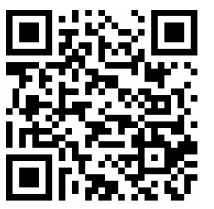

Recibido • Received • Recebido: 02 / 09 / 2016

Corregido • Revised • Revisado: 26 / 02 / 2018

Aceptado • Accepted • Aprovado: 31/ $03 / 2018$

\footnotetext{
${ }^{1}$ El presente trabajo y las investigaciones que le dan respaldo, fueron elaboradas con la tutoría y acompañamiento de la Dra. Teresita Izura quien falleció en 2015. A ella nuestro reconocimiento y agradecimiento afectuoso.

${ }^{2}$ The present work and the studies supporting it were elaborated with the tutoring and accompaniment of Dr. Teresita Izura who passed away in 2015. To her our affectionate acknowledgment and gratitude.

${ }^{3}$ Este trabalho e as pesquisa que deram origem, foram desenvolvidos com tutoria e orientação da Dra. Teresita Izura, falecida em 2015. A ela nosso carinho e agradecimento afetuoso.

${ }^{4}$ Licenciada en Sociología por la Universidad Nacional de Cuyo y Magister en Criminología por la Universidad del Aconcagua, Mendoza, Argentina. Docente de Metodología de Investigación Social en diversas Carreras de grado. Directora de numerosos proyectos de investigación vinculados a las temáticas del género y mujeres, educación superior y privación de libertad. Ha publicado artículos y capítulos referidos a sus incumbencias. Docente de Metodología de Investigación Social en las Carreras de Trabajo Social y Comunicación Social de la Facultad de Ciencias Políticas y Sociales de la Universidad Nacional de Cuyo; y en la Carrera de Niñez, Adolescencia y Familia de la Facultad de Psicología de la Universidad del Aconcagua, Mendoza, Argentina.

${ }^{5}$ Licenciado en Documentación y Gestión de la Información por la Universidad Nacional de Cuyo, Mendoza, Argentina. Especialista en Docencia Nivel Superior por la Universidad Juan A. Maza, Mendoza, Argentina. Docente de Estadística y Bibliometría. Ha sido co-autor de artículo de investigación en la problemática de finalización de tesinas. Docente en la cátedra Estadística y Bibliometría de la Facultad de Educación Elemental y Especial de la Universidad Nacional de Cuyo, Mendoza, Argentina.
} 
doi: http://dx.doi.org/10.15359/ree.22-2.15

URL: http://www.una.ac.cr/educare

CORREO: educare@una.cr

Resumen:Teniendo en cuenta que el ámbito de la educación se encuentra sometido a una permanente dinámica de renovación, resulta de interés este escrito en la medida en que se orienta a describir y analizar cuáles son las dificultades que enfrentan estudiantes que ingresan a dos unidades académicas, una pública y la otra privada, Facultad de Educación Elemental y Especial de la Universidad Nacional de Cuyo y Facultad de Psicología de la Universidad del Aconcagua, cuando entran en contacto con la búsqueda de información, como también, con los tópicos de la denominada alfabetización académica: escritura y lectura. Coincidencias teóricas plantean que existe una elocuente dificultad de adaptación a la vida universitaria, la que se ve agravada, si se tienen en cuenta los trances para el trabajo con herramientas de búsqueda de información. Frente a esta problemática, resulta indispensable considerar las ventajas que podría proporcionar para el estudiantado la incorporación de una nueva cultura académica. Entonces, se constituye en objetivo general de este trabajo, conocer la vinculación que puede establecerse entre las estrategias de búsqueda de información, de lectura y escritura, con la capitalización de conocimientos al inicio del recorrido universitario, para estudiantes de primer año del Profesorado de Grado Universitario en Educación General Básica, de la universidad pública, intentando encontrar puntos de comparación con el grupo que inicia el cursado en las licenciaturas que se dictan en la Facultad de Psicología, de gestión privada: Psicología; Niñez, Adolescencia y Familia, y Criminalística. Para el logro de este, se trabajó con una metodología de diseño estructurado, cuantitativa, en la que se aplicó la técnica de encuesta, administrada con un cuestionario como instrumento de recolección de datos, confeccionado ad hoc para estas investigaciones. La selección de las 146 personas participantes respondió a una estrategia muestral no probabilística, que consideró las dos universidades estudiadas y las cuatro carreras en su interior escogidas. El estudiantado debía estar cursando la mitad del segundo año lectivo, de manera que pudiera dar cuenta de los aspectos a indagar. Los datos obtenidos fueron procesados desde una estadística descriptiva, univariada y bivariada, que brindara una imagen descriptiva de los asuntos estudiados y las principales variables e indicadores mencionados en los objetivos de investigación. Así, a modo de conclusión, respecto al contacto con las fuentes de información, es notable que cerca de un 13\% del estudiantado de ambas universidades no posee TIC para usar en su desempeño universitario, pese a las políticas públicas que en instituciones del Estado se ejecutaron años atrás. Una de cada cinco personas no sabe buscar información académica en internet y desconoce y confunde las herramientas disponibles. En torno a los ejes de la alfabetización académica, lectura y escritura, la falta de lenguaje disciplinar especifico aparece como una de las principales dificultades, tanto para producir como para comprender un texto, acompañada por la ausencia de ejercitación en la producción de textos académicos propios. Pese a la inicial suposición, no se encontraron diferencias significativas atribuibles a la cualidad pública y privada de las universidades de pertenencia de los grupos estudiados.

Palabras claves: Alfabetización académica; lectura; escritura; fuentes de información; estrategias.

Abstract: Considering that education is subject to permanent dynamic renewal, this study aims to describe and analyze what difficulties are facing students enrolled in two different universities: one is public (Faculty of Elementary and Special Education, National University of Cuyo); the other one is private (Faculty of Psychology, Aconcagua University). Students face difficulties when they get in contact with searching information tasks, as well as with the topics of the so called academic literacy. Theoretical coincidences suggest the existence of an eloquent difficulty of adaptation to university life, which is compounded when taking into account difficulties in working with information search tools as for reading and writing are the basic and constitutive academic elements of literacy. In the 
face of this problem, it is essential to consider the benefits that the incorporation of a new academic culture could provide to students. So, the general objective of this study becomes to find the link that can be established between the strategies of information search, reading, and writing, and the capitalization of knowledge at the beginning of the university studies for freshmen students in the Faculty of Elementary and Special Education, University of Cuyo (FEEyE), and the Faculty of Psychology, Children, Youth and Family, and Criminalistics, University of Aconcagua; both are located in Mendoza, Argentine. A structured, quantitative design methodology was implemented to achieve this objective; in this methodology, the survey technique was applied, and a questionnaire was used as a data collection instrument, prepared ad hoc for these studies. The 146 participants selected responded to a non-probabilistic sampling strategy, which considered the two universities under study and the four careers chosen. To apply the strategy the students should be enrolled in the second year of their careers, so that this condition could account for the aspects under research. The data obtained were processed from a descriptive, univariate and bivariate statistics, which would provide a descriptive image of the subjects studied, as well as the main variables and indicators mentioned in the research objectives. Thus, by way of conclusion, regarding the contact with sources of information, it is notable that about $13 \%$ of the students of both universities do not use ICTs in their university performance, despite public policies in state institutions executed years ago. One in five students does not know how to look for academic information on Internet; they confuse and do not know the available tools. Concerning the axes of academic literacy, reading and writing, the lack of specific disciplinary language appears as one of the main difficulties both to produce and to understand a text, accompanied by the absence of exercise in the production of academic texts of their own. Despite the initial assumption, no significant differences were found to be attributable to the public and private qualities of the universities where the groups under study were enrolled.

Keywords: Academic literacy; reading, writing; information sources; strategies.

Resumo: Considerando que o campo da educação está sujeito a uma permanente dinâmica de renovação, este trabalho se torna interessante na medida em que sua proposta é descrever e analisar quais são as dificuldades enfrentadas pelos estudantes que iniciam em duas faculdades, uma pública e uma privada. A Faculdade de Educação e Educação especial da Universidade Nacional de Cuyo e a Faculdade de Psicologia da Universidade de Aconcagua são as instituições que fazem parte deste trabalho e seus estudantes, quando entram em contato com a busca de informações, como também com os temas da chamada alfabetização acadêmica: leitura e escrita. As coincidências teóricas sugerem que existe uma dificuldade eloquente na adaptação à vida universitária, que é grave quando existem dificuldades para trabalhar com ferramentas de busca de informação. Confrontado com este problema, é essencial considerar os benefícios que poderiam ajudar aos universitários a incorporação de uma nova cultura acadêmica. Então, o objetivo geral deste trabalho é conhecer o vínculo que pode existir entre as estratégias de busca de informações, de leitura e de escrita, com a organização do conhecimento ao iniciar o curso universitário, para estudantes do primeiro ano de Licenciatura em educação na faculdade de educação, da universidade pública, tentando encontrar pontos de comparação com o grupo que começa os estudos de licenciatura na Faculdade de Psicologia, na universidade privada: Psicologia: infância, adolescência, família, e criminalística. Para efetuar este trabalho, foi utilizado um desenho metodológico estruturado e quantitativo, no qual foi aplicado a técnica de entrevista; os dados foram conseguidos através de um questionário, 
doi: http://dx.doi.org/10.15359/ree.22-2.15

URL: http://www.una.ac.cr/educare

CORREO: educare@una.cr

\begin{abstract}
elaborado ad hoc para esta pesquisa. A seleção das 146 pessoas participantes respondeu a uma estratégia de amostragem não aleatória, considerando as duas universidades participantes e as quatro carreiras escolhidas. Os estudantes universitários tinham que estar cursando a metade do segundo ano acadêmico, de modo que poderia dados aos aspectos que seriam investigados. As informações obtidas foram processadas a partir de estatística descritiva, uni-variada e bivariada, que iria fornecer um quadro descritivo dos temas estudados, as principais variáveis e indicadores mencionados nos objetivos da pesquisa. Portanto, como conclusão, relacionado com o contato com as fontes de informação, aproximadamente $13 \%$ dos estudantes de ambas as universidades não têm TIC para usar em seu desempenho acadêmico, apesar de haverem sido executadas fazem anos atrás, nas políticas públicas nas instituições estatais. Uma em cada cinco pessoas não sabem buscar informações acadêmicas na internet, desconhecem e confundem as ferramentas disponíveis. Em torno aos núcleos da alfabetização acadêmica, leitura e escrita, a falta de uma linguagem específica da matéria aparece como uma das principais dificuldades, tanto para produzir como para compreender um texto, acompanhada da ausência de exercício em relação a produção de textos acadêmicos. Apesar da suposição inicial, não houve diferenças significativas atribuíveis à qualidade das universidades públicas e privadas nos dois grupos estudados.
\end{abstract}

Palavras-chave: Alfabetização acadêmica; leitura; escrita; fontes de informação; estratégias.

\title{
Introducción
}

Como han señalado diversos estudios (Caldera y Bermúdez, 2007; Carlino, 2009; Lozano, 2010; entre otros), la educación asiste continuamente a cambios profundos, se modifican los paradigmas que la estructuran y las herramientas involucradas en el proceso de aprendizaje, se registran innovaciones en el plano del acceso a la información y se actualizan los currículos de las carreras, de modo que todo parece estar constantemente en movimiento.

Así pues, dos conceptos centrales movilizan la indagación que se llevó adelante y que da origen a la reflexión que aquí se inicia. Por un lado, la alfabetización en la universidad y, por otro, el contacto con las fuentes de información, constituidos en los ejes articuladores de la investigación desarrollada en dos momentos, en el ámbito de la Universidad Nacional de Cuyo (UNCuyo), institución pública, a lo largo del año 2012, y en 2014 al interior de la Universidad del Aconcagua (UDA), de gestión privada; ambas con sede en Mendoza, Argentina.

El estudio que se llevó a cabo parte de la siguiente pregunta de investigación: ¿Cómo se vinculan las estrategias de búsqueda de información, de lectura y escritura con la capitalización de conocimientos, para estudiantes que dan inicio al recorrido universitario, y que caracteres tiene? Tanto para el caso de la carrera de Profesorado de Grado Universitario en Educación General Básica (también llamado en Educación Primaria), Facultad de Educación Elemental y Especial, como de las licenciaturas en Psicología, Niñez, Adolescencia y Familia, y en Criminalística. Será a partir de este interrogante que se desentrañará la complejidad que las variables mencionadas importan. 
Se constituyó en objetivo general del estudio: conocer la posible vinculación entre las competencias previas y el conocimiento de estrategias de búsqueda de información, de contacto con bibliografía en diferentes formatos, y hábitos de lectura y de escritura, como estrategias favorecedoras de la capitalización de aprendizajes, en el inicio del recorrido en el ámbito universitario respecto de estudiantes de las carreras seleccionadas.

De manera trasversal, se intenta, en todo momento, averiguar si existen divergencias entre los ámbitos públicos y privados de educación superior, sobre los tópicos de interés centrales.

Luego de identificar, desde el rastreo bibliográfico y los contactos previos con personal experto, los principales vacíos en los conocimientos existentes, el proceso de investigación buscó describir, por un lado, los obstáculos que manifiesta el estudiantado ingresante a la hora de entrar en contacto con bibliografía, sea de manera directa y simple (indagar en una biblioteca o en un ejemplar de un libro) como así también, a la hora de utilizar herramientas informáticas que pueden proveer de información. Por otro lado, las dificultades que mencionan estudiantes ingresantes respecto las estrategias de lectura y escritura necesarias al incorporarse en la denominada cultura académica. Finalmente, también conocer cuáles son los aspectos que favorecen al estudiantado universitario en el manejo de las fuentes de información existentes, como así también en los ejes del proceso de aprendizaje.

El estudio empírico facilitó la medición de las variables establecidas y permitió dar cuenta de los preceptos planteados, los cuales se organizaron en torno a tres ejes: fuentes de información, competencias de escritura y competencias de lectura.

La estrategia metodológica utilizada para el abordaje del tema fue de tipo cuantitativa y se aplicó la técnica de entrevista estructurada o cuestionario para la recolección de datos, cuyos resultados se analizaron con algunos recursos de estadística descriptiva.

Se trabajó con una muestra no probabilística enmarcada en un diseño exploratorio y descriptivo, para buscar identificar variables que permitieran contrastar las hipótesis de trabajo formuladas, a saber:

- El estudiantado ingresante a la vida universitaria cuenta con serias dificultades y falencias en el conocimiento y manejo de estrategias de búsqueda de información, ya sean tradicionales o virtuales.

- Las competencias de escritura y lectura son elementos considerados bisagras en las estrategias de acrecentamiento de los aprendizajes en el inicio de la vida universitaria $y$, sin embargo, el estudiantado denota profundas carencias al respecto. 
doi: http://dx.doi.org/10.15359/ree.22-2.15

URL: http://www.una.ac.cr/educare

CORREO: educare@una.cr

\section{Aspectos teóricos}

A fin de dar cuenta del posicionamiento teórico conceptual asumido en esta investigación, se proceden a desarrollar aquellos constructos que se consideran centrales a esta misma, aun cuando pudieran resultar conocidos o intuitivamente cercanos. Si bien es varia la bibliografía que existe sobre estos tópicos, no lo es cuando de estudios locales se trata, ni para la especificidad del abordaje de las trayectorias universitarias, como aquí se propuso. Por lo tanto, ha sido en torno a un estricto grupo de trabajos con base en los que se construyó el marco teórico de la investigación; selección que responde a su empatía con el contexto inmediato y a que contaran con trabajo empírico o de campo, en cierto modo equiparables al recorte específico realizado.

\section{Cultura y alfabetización académica, sentidos y alcances}

Para iniciar el recorrido teórico es necesario apelar a nociones íntimamente imbricadas con el fenómeno en estudio, empezando por una autora coterránea, que ha dedicado mucho de su trabajo a la temática y con la que este quipo comparte, de modo absoluto, propuestas y perspectivas. Así, es valioso partir del concepto de cultura académica, configurada, según Carlino (2009), por las representaciones y las prácticas institucionales que con cierta continuidad temporal se vienen llevando a cabo en un espacio determinado, y alude a aquellos saberes $y$ haceres propios de una particular comunidad académica.

Una peculiaridad de este entramado de pensamiento y acción compartidos es que no suele ser cuestionado al interior de la comunidad para la cual resulta de algún modo funcional, en la medida en que esta trama confiere al conjunto un carácter pseudo-natural y necesario. (Carlino, 2009, p. 6)

La cultura académica es una resultante y sintetiza lo que se ha decantado en el proceso de cimentación de los conocimientos científicos, es decir, en el trabajo de construcción, apropiación y ampliación de estos. En este ámbito, la comprensión y la producción de textos se entienden supeditadas a la construcción de los conocimientos, teniendo en cuenta que los conocimientos académicos, por lo general, se formalizan en textos y esto exige un ejercicio de su interpretación y de saberes respecto a las formas de circulación del conocimiento propio de cada área disciplinar.

Frente al hecho de que tanto la producción como la circulación de conocimientos son estructuradas y formalizadas de acuerdo con cada campo disciplinar, se encuentra la condición de alfabetización como aspecto indispensable para participar de estos procedimientos en cada una de las determinadas comunidades científicas. 
No obstante, la realidad de esta afirmación nos lleva también a pensar en la dificultad que surge cuando se pretenden currículos multidisciplinarios o transdisciplinarios, común en muchas carreras de grado y más aún en las de posgrado. Son grandes las dificultades que desde el estudiantado se expresan al no encontrar cierta coherencia entre los vocabularios que en las materias se van proponiendo, pensando en cuerpos docentes que provienen de diversos ámbitos de formación y no de la carrera misma en que se desempeñan; ganar en riqueza de aportes conlleva el costo de intentar una serie de líneas básicas vinculadas a esta formación integral y transversalizada.

El ingreso de una persona a la vida como estudiante de universidad implica su inmersión en aquel recorte disciplinario que eligió a fin de formarse académicamente, expresada por Carlino (2009) en toda su profundidad:

La incursión de un inmigrante a una cultura nueva, con todas las características del visitar y vivenciar valores y normas de comportamiento específicas y particulares de la cultura de esa comunidad, y de la que hay que aprender para poder interactuar en ella correctamente, hasta lograr ser miembro de la misma apropiándose de esa cultura. (p. 6)

En esta incursión, el estudiantado debe no solo producir y leer escritos con el fin de aprobar las instancias de evaluaciones parciales y finales, sino también con destino a la participación en otras actividades propias de la vida académica como presentación de monografías, informes de lecturas, sistematización de experiencias, presentaciones y ponencias en congresos y redacción de artículos, entre otras posibles.

Atendiendo a los aportes teóricos consultados, es posible destacar con claridad dos ejes centrales al interior de la cultura académica, a saber: la lectura y la escritura:

Se constituye la lectura como fundante del conocimiento, del aprendizaje de cualquier contenido curricular y de la posibilidad de tomar contacto con la producción académica de una disciplina. Sin embargo, la lectura no puede considerarse de manera aislada, dado que también la escritura se estructura como un eje que colabora con la función epistémica de la lectura.

Carlino (2009) describe a las personas que ingresan a estudiar a la universidad como pasivas frente a los textos que en cada materia se les indica leer, desprovistas de metas propias frente a esa actividad, y sin saberes acerca de los contenidos que dichos textos portan. Quienes nos desempeñamos en el hacer docente, tenemos experiencia numerosa en este sentido, tanto al observar las prácticas de nuestros grupos sucesivos de estudiantes, como al reflexionar sobre las propias; y las propuestas y consignas que para ellos elaboramos.

Los inconvenientes que presenta la tarea son tan complejos que se hace indispensable la presencia docente para acompañar esta actividad y asumirla como responsabilidad propia y 
doi: http://dx.doi.org/10.15359/ree.22-2.15

URL: http://www.una.ac.cr/educare

CORREO: educare@una.cr

primordial, no accesoria (Goyes y Klein, 2012). La lectura, además, es una actividad que contiene y requiere de procesos selectivos que le son propios y que cada lector va privilegiando de acuerdo con la intención u objetivo, lo que, según la afirmación hecha, en este grupo se vería limitado.

En relación con el otro aspecto destacado, la escritura, la autora considera que esta actividad es nodal en las trayectorias universitarias, acepta que no se aprende allí a escribir neutralmente, sino en medio de la problemática propia de cada disciplina. Así como escribir, leer y pensar son actividades que aparecen estrechamente vinculadas en la cotidianeidad universitaria, aparentemente condicionando la comprensión de lo que las personas estudian; en el mismo sentido la articulación sería absoluta entre comprensión, producción de textos y conocimiento (Carlino, 2009).

Entonces, lo que se pretende es que el estudiantado arbitre los medios necesarios para que la escritura y lectura sean el basamento de análisis, argumento y conclusión, a efectos de llegar a ser partícipe de la lógica discursiva de las diversas disciplinas educativas con las que les corresponde sortear en la vida universitaria (Goyes y Klein, 2012).

Carlino (2009), en este sentido, plantea algo que en el imaginario tiene mucha presencia, y es la concepción generalizada de la escritura como un mero medio de expresar lo que se piensa y de transmitir conocimiento, como así también, la consideración de que esta competencia se aprende antes de ingresar a la universidad. Por lo tanto, a fin de mejorar las carencias detectadas, las acciones detectadas en las instituciones tienden a ser oportunidades de ejercitación, desarticuladas entre sí, no trasversales, y mucho menos consideradas como parte de una práctica social (Carlino, 2013).

Sin embargo, numerosas investigaciones -en el ámbito de la antropología, didáctica, historia y psicología - y la propia experiencia docente han demostrado lo contrario: la escritura posee potencial epistémico, es decir, no solo es un medio de registro y comunicación, sino que es factible de constituirse como instrumento para sistematizar, desarrollar, revisar y transformar el propio saber (Carlino, 2009).

Por tanto, teniendo en cuenta la relevancia que puede asignársele a la escritura, dado que a lo largo de la formación universitaria constituye una herramienta fundamental, es importante visualizar todos los aspectos implicados para una persona estudiante cuando se enfrenta a un texto académico, sea para producirlo o para leerlo.

En el contexto concreto de Argentina, donde las universidades públicas aglutinan gran porcentaje de la comunidad universitaria nacional, es imperioso considerar en primer plano lo relativo a las trayectorias previas diferenciales que cada ingresante porta, no solo desde el punto de vista de la educación formal, sino también, laboral, social y económico. 
Como corolario, destaca la tesis central del trabajo de Carlino (2009), en cuanto expresa "no se trata solamente de que ellos llegan mal formados de sus estudios secundarios previos; se trata de que al ingresar a la formación superior se les exige un cambio en su identidad como pensadores y analizadores de textos" (p. 10).

Como cavilación de este equipo, surge atender la tensión que aparece entre esta necesidad de cambio de identidad frente a la lectura de un texto; y la ausencia de espíritu crítico que de algún modo implica lograr una exitosa culturización académica, en tanto esta no prevé la posibilidad de reflexión acerca del conjunto de pautas explícitas y tácitas que esta conlleva. Es decir, en este proceso de inserción a la cultura universitaria, lo que por un lado se niega, por otro aparece como deseable.

Teniendo en cuenta el eje conceptual que guía esta elaboración, es importante avanzar sobre otro concepto estrechamente involucrado con lo hasta ahora desarrollado: el de alfabetización académica. Para Caldera y Bermúdez (2007) como para Carlino (2009), la lectura y la escritura son fundamentales en la producción, posesión y difusión del conocimiento. Por lo tanto, es necesario considerar, en las instancias de educación superior, el concepto de alfabetización académica, entendida como una suerte de síntesis que conjuga estrategias y actividades de lectura y escritura, propias de cada disciplina.

Por ello, estos autores proponen integrar el trabajo al interior de cada cátedra, combinando los aprendizajes disciplinares con la enseñanza de lectura y escritura de textos académicos; para que, de esta manera, el estudiantado esté en mejores condiciones de acceder, comprender y producir material propio del campo de conocimiento científico en que se insertan.

Sin embargo, según Caldera y Bermúdez (2007), la práctica educativa habitual se encuentra alejada de esta propuesta, y se caracteriza por inducir procesos de memorización, reproducción, fragmentación y acumulación del conocimiento, con lo que se retrasan los procesos de aprendizaje y se contribuye a la disminución de la autonomía en el uso del lenguaje. Resulta una observación conjunta de una variedad de especialistas, el hecho de que docentes de las asignaturas se programan para enseñar conceptos disciplinarios específicos, obviando la responsabilidad que pudiera caberles respecto la enseñanza de la lecto-escritura. Debido a que esta tarea debería presentar integralidad y trascender más allá de los contenidos conceptuales, se espera que los cuerpos docentes impartan instrucciones para el desarrollo de estrategias de comunicación y para la promoción de actitudes que impulsen al alumnado a aprender durante toda la vida. Para ello sería fundamental integrar destrezas en este sentido, combinándolas con los contenidos propios de cada asignatura, y no solo al inicio de los recorridos, como suele ponerse en práctica (Goyes y Klein, 2012).

En Argentina, al considerar el concreto caso de los currículos de carreras profesionales no docentes, en su mayoría las parrillas no dan cuenta de espacios que tengan que ver con 
doi: http://dx.doi.org/10.15359/ree.22-2.15

URL: http://www.una.ac.cr/educare

CORREO: educare@una.cr

aportes a la formación pedagógica de quienes egresen; es decir, gran parte de la docencia universitaria y de nivel medio no cuenta con las herramientas para llevar a cabo ese trabajo transversal, y mucho menos si de colectivos específicos se trata (Torino, 2012). Estas quedarán supeditadas a posteriores formaciones, vocación o aptitud individual, ya que de ningún modo son garantizadas por la titulación que se ha obtenido.

Agravando esta afirmación, desde un enfoque denominado epistemológico cognoscitivo, el individuo no es pasivo en el proceso de enseñanza aprendizaje, sino que es partícipe en un proceso caracterizado por ser activo, dinámico, dependiente del tipo de información que se maneja, influido por él mismo, en tanto aprendiz y constructor de significados. Allí, el personal docente debería ser responsable, en tanto mediador de estrategias cognitivas como metacognitivas, es decir, debe darle espacio específico, en el aula, a la generación de procedimientos cognitivos complejos, para poco a poco traspasar al alumnado el control de esa responsabilidad (Caldera y Bermúdez, 2007).

Este punto de vista promueve aprendizajes significativos que integren los nuevos conocimientos, paulatinamente, con el cuerpo de saberes que la persona ya posee, para generar una suerte de encastre entre lo que se va adquiriendo y aquello con lo que cuenta.

También existen, para esta perspectiva, estrategias metacognitivas que deben integrarse al proceso. Estas implican la toma de conciencia del sujeto que aprende acerca de sus propios procesos y niveles de conocimiento. Por ello, propone trabajar en la concientización y control de estos, por medio de estrategias de planificación, regulación y evaluación del procesamiento de la información (Caldera y Bermúdez, 2007).

La instancia de la clase cuyo modo sea meramente expositivo con la pretensión de instruir, transmitir contenidos aislados de la realidad y desarticulados ha de modificarse, para dar lugar en ella al intercambio de experiencias e ideas, al incentivo de valores y a constantes reflexiones, en la perspectiva de una la formación integral de las personas. El personal docente debe facilitar situaciones que permitan pensar, discernir, reflexionar, expresarse con criticidad y creatividad, y que de ningún modo se agoten en lo disciplinar; por lo tanto, consideramos que el cuerpo docente también debe ser un sujeto receptivo en los procesos de alfabetización académica (Torino, 2012).

En este sentido, años después, Carlino (2013) se encuentra trabajando en una actualización del concepto de alfabetización académica, corriendo el eje de la responsabilidad del proceso del estudiantado, para incorporar las instituciones y los cuerpos docentes a lo largo de todo el trayecto académico y de modo contextualizado, tan es así, que pregona las alfabetizaciones académicas, haciendo énfasis en su pluralidad. 
Sin duda, la alfabetización académica no es una habilidad que se logra de una vez y para siempre. Esto cuestiona la idea de aprender a producir e interpretar el lenguaje escrito como un asunto asegurado y concluido al incorporarse a la educación superior. Pertenecer a la cultura académica implica la comprensión y producción de textos, y ser una persona alfabetizada en las prácticas discursivas surge como la condición necesaria para participar con pertinencia en estas comunidades que utilizan el lenguaje con determinados modos y propósitos.

\section{Las competencias informacionales en el ámbito de la educación superior}

Ahora bien, como la intención de este trabajo es establecer vinculación entre conceptos asociados con el fin de desentrañar la compleja situación que significa el aprendizaje del estudiantado en la educación superior, se esbozan, a continuación, una serie de apreciaciones contundentes respecto del contacto del estudiantado con las fuentes de información y, más específicamente, con las competencias informacionales.

Ciertalínea de estudios, queeste equipocompartey revisa, sostienequeestas competencias estarían representadas por condiciones previas o modalidades que hacen posible la actuación relativa a la información por parte del estudiantado universitario, particularmente, en torno a las experiencias de acceso, tratamiento, apropiación y comunicación de la información (BarbosaChacón, Barbosa-Herrera, Marciales-Vivas, Castañeda-Peña, 2010).

La línea de investigación seguida pretende superar la perspectiva de un alfabetismo en sentido instrumental, orientándolo hacia una mirada sobre los modos de interacción con la información y su consumo, especialmente de aquellos aspectos que intervienen, condicionan y transforman dicho hacer (Marciales-Vivas, Gonzále-Niño, Castañeda-Pena y Barbosa-Chacón, 2008). Así pues, desde el concepto integral de competencias informacionales propuesto por Barbosa-Chacón et al. (2010), las relaciones con las fuentes de información y, en especial, la apropiación de conocimiento, responden a tres tópicos, a saber: características de orden individual, factores vinculados a la historia personal y aspectos contextuales y culturales.

Esto puede resultar una verdad de Perogrullo. En el contexto de países insertos en la América latina, estos factores, sin duda, determinan y configuran todo tipo de trayectoria de educación formal. Sociedades caracterizadas por la polarización o desigualdad de las clases o sectores sociales dan por resultado experiencias de gran diversidad que posicionan, de maneras múltiples, a cada persona frente al leer, escribir e informarse. Esto adquiera gran relevancia en Argentina, si tenemos en cuanta la alta presencia de la escuela y universidad pública, que aglutinan un estudiantado heterogéneo y diverso, en sentido socioeconómico.

Ciertamente, los diferentes entornos donde transcurren las historias de vida de aprendices $y$, por supuesto, docentes dan sentido a las destrezas construidas por estos grupos para la 
doi: http://dx.doi.org/10.15359/ree.22-2.15

URL: http://www.una.ac.cr/educare

CORREO: educare@una.cr

apropiación y uso de las fuentes de información, y explican muchas de las prácticas que se evidencian en los espacios académicos (Marciales-Vivas et al., 2008).

Según estos estudios, luego de recopiladasy analizadas, es posible identificar trestendencias que caracterizan las definiciones relacionadas con competencias. La primera invita a considerar la competencia como habilidad, destaca que para su desarrollo intervienen la enseñanza y la práctica, vinculada así a desempeños propios del orden académico. La segunda considera la competencia como una habilidad para el acceso a la información, como el desarrollo de un hacer de orden instrumental que permite ese acceso. Y la tercera entiende esta competencia como una práctica con dimensión social, para que la gente pueda funcionar en sociedad.

De allí que la atención se centre en la relación que existe entre el desarrollo de las competencias informacionales y la formación de un sujeto social, capaz de asumir con conciencia, tanto crítica como ética, la diversidad y complejidad de factores que median el acceso a la información. (Marciales-Vivas et al., 2008, p. 647)

Si bien puede parecer tedioso recorrer a cada una de ellas, resulta sorprendente ver cómo aún es factible, para muchas líneas de pensamiento, sostener cierta independencia de estos procesos educativos respecto a los ambientes sociales previos y circundantes. Más aún, ante la cruda avanzada de gobiernos neoliberales en la región latinoamericana, impulsadores de políticas educativas mercantilistas que enarbolan con liviandad ideas meritocráticas, en estos días es valiosa la reflexión.

Marciales-Vivas et al. (2008) afirman, en su trabajo, que asumir la primera o segunda postura implica excluir la consideración acerca de que el desarrollo de esas habilidades está relacionado íntimamente con la trayectoria de vida de las personas, entendida esta desde su previa interacción con fuentes de información y con personas significativas para esas instancias.

Asimismo, la no consideración del sujeto como ser social les significa dejar de lado los efectos que sobre el contexto provoca la relación sujeto competente-fuentes de información, en tanto instancia con oportunidad de constituir una persona informada y participativa en procesos de decisión con repercusión colectiva.

Estaúltima afirmación permite introducirel aporte de Ferreiray Dudziak (citado por MarcialesVivas, 2008), quienes incorporan al debate la idea de ciudadanía plena, a partir de sostener que la incorporación de los sujetos a procesos de decisión estará condicionado por las competencias informacionales que los caractericen, inscribiéndose, sin dudas, en la tercera perspectiva.

Así, pues, aportan la dimensión histórica de las competencias informacionales, en la medida en que cuando se piensa en quién usa la información se visualiza un "sujeto dinámico y cambiante" (Ferrerira y Dudziak, citado en Marciales-Vivas et al., 2008, p. 647). 
Las categorías teóricas que las autoras proponen dan un nuevo marco al tratamiento de las competencias informacionales, donde, si bien la alfabetización digital y los procesos cognitivos sostienen su centralidad, es pertinente incorporar las articulaciones posibles entre habilidades, conocimientos y valores históricamente edificados por cada sujeto, al forjarse como informacionalmente competente. Aspectos que desde un punto de vista sociológico nunca podrían dejarse de lado, retomando lo pertinente respecto de la educación universitaria pública argentina.

Avanzando en la revisión teórica del asunto, es importante presentar una nueva faceta para la comprensión profunda de las competencias informacionales.

Para Alvarado, el sujeto actuante, que usa las fuentes de información, se constituye, como tal, en cuanto actúa modalizado por diferentes condiciones: si es por un saber hacer, indica que dispone de competencia cognitiva; o si es por un poder hacer, da cuenta de una capacidad; cuando está modalizado por un querer hacer, significa que tiene voluntad para actuar; o por un deber hacer, es decir, dispone de una prescripción. Finalmente, cuando es modalizado también por sus creencias, significa que ha asumido los determinantes -tanto de su cultura como de su grupo social de referencia- para orientar su acción. (Marciales et al. en Barbosa-Chacón et al., 2010, pp. 135-136)

De esta manera, para las autoras (Marciales et al. en Barbosa-Chacón et al., 2010), la competencia informacional de quien es estudiante no se encuentra definida solamente por lo que hace o lo que es frente a la información, sino que tiene implicancias más profundas, en la medida que sus creencias, adhesiones, prescripciones, voluntades, motivaciones, capacidades y saberes definen sus potenciales competencias; lo que, a su vez, inexorablemente, está relacionado con sus experiencias de vida y, también, con su condición social. Entonces, al considerar estos nuevos aspectos, lo relativo a la relación de las personas con la información posee una serie de dimensiones psicológicas individuales, que complejizan su estudio y, por supuesto, su descripción y medición.

Cabe cuestionarse acerca de una de las carencias que desde el primer momento acompaña el proceso de reconceptualización propuesto por Barbosa-Chacón et al. (2010): ¿cuáles son las maneras de aproximarse a la observación o medición de las competencias informacionales? Si bien los autores se comprometen a plantear una estrategia de observación que tenga como horizonte dar cuenta de dicha competencia de manera aproximada y articulada, en el escenario de la educación superior no ha sido posible encontrar referencia de ello.

Ahora bien, un gran desafío queda abierto, en tanto afirman que es "responsabilidad ... de las organizaciones educativas ... la generación de estrategias, mecanismos y políticas para caracterizar las prácticas de uso de las fuentes de información" (Barbosa-Chacón et al., 2010, p.123), como también propender al incremento de habilidades o competencias para su 
doi: http://dx.doi.org/10.15359/ree.22-2.15

URL: http://www.una.ac.cr/educare

CORREO: educare@una.cr

tratamiento y apropiación por parte del estudiantado; considerando siempre el marco en que se da el proceso de enseñanza aprendizaje y la etapa evolutiva personal de cada una de ellas.

En vistas a este desafío, el trabajo de Barbosa-Chacón et al. (2010) intenta contribuir con dos problemáticas propias de la educación superior, lo que podría ser compartido respecto el estudio que aquí se desarrolla: incrementar el saber en torno a la relación de la diada compuesta por educandos-fuentes de información; y relevar obstáculos que se presentan en la adquisición de competencias básicas respecto a su uso.

Finalmente, es valioso hacer mención de otros actores que surgen en la temática y que hasta ahora no fueron mencionados. Si se entienden como competencias informacionales al conjunto de conocimientos, habilidades, disposiciones y conductas que capacitan a los individuos a efectos de poder reconocer cuándo necesitan información, dónde localizarla, cómo poder evaluarla y cómo poder darle el uso adecuado en virtud del problema que se les presenta (Varela, 2009) y, según ya se ha avanzado, podría hablarse de una tarea conjunta enmarcada entre la docencia y la biblioteca con un objetivo concreto y claro: fomentar, en quien es estudiante, la manera de integrar conocimientos, habilidades y actitudes. Serán, pues, tanto el personal docente como de bibliotecología quienes deberán aunar esfuerzos para brindar competencias en gestión de la información, a efectos de que sirvan para el autoaprendizaje de la persona estudiante.

Es consecuente retomar aquello expresado en torno a la falta de especificas herramientas para la alfabetización académica que poseemos quienes nos desempeñamos como docentes en el contexto universitario argentino; ya que ahora se debe sumar a ese vacío el relativo a la alfabetización digital y las propias competencias informacionales de quien ocupa el lugar de maestro o maestra.

\section{Las nuevas tecnologías y el proceso de aprendizaje: Constantes retos}

Recorriendo los edificios históricos de la universidad en la región, como también los edificios modernos, tanto desde la arquitectura como desde las imágenes que cada aula arroja y los testimonios, no hay margen de duda respecto a que la tradición en educación superior ha respetado una lógica particular apoyada durante décadas en un modelo de enseñanza donde el estudiantado asiste a las clases del profesorado, este imparte conocimientos desde una modalidad expositiva por excelencia, el estudiantado toma apuntes y con posterioridad avanza en la tarea de comprensión y memorización de la bibliografía indicada por su docente con el fin de poder superar las instancias de evaluación planteadas.

Es en este esquema estancado y reiterativo, en el cual irrumpen las denominadas tecnologías de la información y comunicación TIC, representando un reto en los procesos y métodos habituales de enseñanza y constituyéndose, por tanto, en la oportunidad de una auténtica revolución en el marco de la pedagogía. 
El fomento del uso de las TIC, tan difundido en el marco de la educación media y superior, pretende que quienes estudian se familiaricen con la adquisición de habilidades transversales relacionadas con conocimientos específicos; intenta establecer un vínculo que permita al estudiantado gestionar información de diversa índole y convertirla luego en conocimiento.

Si bien el actual estudiantado ingresante universitario es nativo digital y experto en el uso de nuevos sistemas y tecnologías de información y comunicación, se le presentan grandes dificultades cuando tiene que enfrentar la desbordante información que las propias TIC proporcionan y llegarlas a convertir en información que pueda preciarse de ser nominada como conocimiento pertinente al ámbito académico. Tareas de búsqueda, selección, ponderación, lectura, comprensión y reproducción de información académica científica resultan de gran dificultad para el estudiantado que hoy transita estudios de nivel universitario.

Por ello, el desafío consiste en que las universidades innoven no solo su equipamiento y recursos en tecnología (desafío surgido y parcialmente alcanzado hace un par de décadas atrás), sino también sus concepciones y prácticas pedagógicas, es decir, modificar el modelo de enseñanza universitario en su globalidad. Quizás, en este marco, las redes puedan realizar aportes para la construcción y desarrollo de un modelo de enseñanza flexible, preponderando la actividad y la posibilidad de generar nuevo conocimiento por parte de cada estudiante (Varela, 2009).

Un ejemplo elocuente lo constituye la incorporación de la telemática en la educación, que tiene su inicio en la etapa preescolar y llega a la universitaria, configurando redes de aulas o círculos de aprendizaje en donde el saber tiene su impronta en compartir información y recursos de un campo científico y tecnológico, aquí se destaca el aprendizaje a distancia, por intermedio de cursos on-line a través de un sistema electrónico sincrónico y asincrónico, donde la persona incorpora conocimiento con recursos con los que cuenta la red.

Así mismo, respecto a internet, el desafío pedagógico se presentaría ante las innumerables plataformas virtuales aprovechables que permiten que aulas, repositorios y bibliotecas se presenten continuamente utilizables, produciendo una suerte de explosión en la disponibilidad de información, donde necesariamente entran en juego otras competencias por parte de los actores involucrados en los procesos de enseñanza y aprendizaje.

Esto sugiere una suerte de reconversión del rol docente que le implicaría dejar de ser emisor o productor de información, para convertirse en un ente tutor o guía en los procesos de búsqueda y selección de información y, desde allí, acompañar el proceso de aprendizaje del estudiantado. Desde allí, las TIC permiten incrementar y multiplicar las instancias de comunicación entre el cuerpo docente y el alumnado, incluso desligándolas de las nociones habituales de tiempo y espacio. 
doi: http://dx.doi.org/10.15359/ree.22-2.15

URL: http://www.una.ac.cr/educare

CORREO: educare@una.cr

En este sentido, con la aparición de las herramientas e-learning, la enseñanza se produce mediante dos tipos de interacción. Por un lado, está la interacción sincrónica que es la que se enfoca en la tutoría, el único recurso con que cuenta es el chat o videoconferencia; en tanto que con la interacción asincrónica, permite estar en tiempo y lugar diferentes, a través de los foros, wikis y correo electrónico (Varela, 2009).

La utilización de plataformas de e-learning en la educación demanda un aumento de la autonomía del alumnado, esto quiere decir que las TIC en el contexto de la educación superior exigen un modelo educativo caracterizado, entre otros rasgos, por el incremento de la capacidad de decisión del alumnado sobre su proceso de aprendizaje (Varela, 2009).

Las TIC ofrecen a las personas usuarias una cantidad desbordante de recursos que otorgan velocidad, simultaneidad, inmediatez, ahorro de tiempo y espacio, cúmulo de información; pero, para que el alumnado maximice el uso de esas nuevas tecnologías, tiene que estar acompañado por un rol de gestoría que lo guíe en el camino de la experimentación y aprendizaje.

Los aportes docentes resultan fundamentales en cuanto a estrategias de búsqueda, capacidad de selección, competencias para la correcta interpretación y contextualización de la información encontrada. Y aquí, también la biblioteca tradicional puede jugar un rol preponderante, ya que, por ser una fuente de información principal, debe asumir la función de ser conductora y asesora en lo que se refiere a la adquisición de competencias en información.

En este sentido, Quindemil (2010) aporta que las aptitudes para el acceso y uso de la información constituyen la base para el aprendizaje continuo a lo largo de toda la vida, implican la posibilidad de apropiarse, asumir, dirigir su propio aprendizaje y de integrarse a la cultura; este aspecto conlleva al estudiantado a una búsqueda de nuevas vías para una formación plena.

Como se ha sostenido en varias ocasiones a lo largo de este trabajo, las universidades enfrentan, en este aspecto, otro desafío: reflexionar sobre sus prácticas docentes e involucrar la formación en aquellas competencias que le permitan incorporar, a la comunidad educativa, la utilización de estas nuevas herramientas, tanto a estudiantes como profesorado. Sin dudas, asumiendo que las formas de enseñanza y aprendizaje se han transformado, a partir de tareas exploratorias probar nuevas estrategias, sin necesidad de buscar un modelo unívoco, sino plantear diversos canales a los que podrá ir adhiriéndose cada quien según sus propias competencias.

No obstante, como ya se propuso, un vistazo a las aulas de grado y posgrado argentinas no refleja ni remotamente la explosión tecnológica referida; casi como si la academia hubiese permanecido ajena a ese proceso global. Pizarras, y en ocasiones equipos multimedia con presentaciones en PowerPoint, se constituyen en la postal más habitual de las aulas de nivel superior, junto con la incomodidad docente ante el uso semioculto de celulares y tabletas, que no logran incorporar a su quehacer. 
Sin dudas existen, como explicación, diferencias generacionales, pero también prejuicios y miedos provenientes de la falta de capacitación del cuerpo docente en el uso e incorporación de las TIC a su labor. Por lo tanto, desde la mirada reflexiva que ejercitamos, el desafío y el compromiso por cerrar esa grieta corresponde a quienes actualmente nos desempeñamos en el lugar de transmitir saberes al estudiantado, y al llevar a cabo desarrollos teóricos sobre el tema debemos, obligadamente, considerar las dos partes de la diada.

\section{Aportes por revisar ante una propuesta educativa de alfabetización informacional}

Así como oportunamente se desarrolló la categoría de alfabetización académica, es momento de introducir un concepto específico: la alfabetización informacional (denominada ALFIN). Esta, como proceso mediante el que una persona aprende a usar la información, obtiene la capacidad de saber cuándo y por qué necesita información, dónde encontrarla, y cómo evaluarla, utilizarla y comunicarla.

Existen a nivel internacional una serie de interrogantes sobre cómo operativizaryllevar adelante las estrategias de alfabetización informacional. A partir del rastreo de cómo se van desarrollando estos temas y las estrategias en relación con diversas propuestas existentes, es posible sistematizar autorías y organizaciones que lideran la aplicación de ALFIN en diversas regiones del globo.

Así, pues, inicialmente, en EEUU en el año 2000 se elabora un documento con cinco normas o resultados a alcanzar en lo que se refiere a la alfabetización informacional, en donde cada norma tiene indicadores y resultados por medir y su aporte esencial radica en la aproximación meta cognitiva a la capacidad de autorregular el aprendizaje. Este punto permite planificar estrategias que se han de utilizar en cada situación, la forma de aplicarlas y controlarlas en el proceso, a fin de evaluarlo y detectar posibles fallos que aparezcan. El proceso meta cognitivo permite su aplicación de forma recurrente, en donde se exige a la persona estudiante que vuelva a una etapa anterior del proceso que está analizando y que revise el planteamiento de búsqueda de información. "La experiencia vital del alumno es fundamental, al servir como base para acceder a otras experiencias superiores" (Quindemil, 2010, p. 102).

Por otra parte, en el Reino Unido, la experiencia realizada en el 2001 establece normas para un modelo de habilidades en información en educación superior, estableciendo que, a través de siete pilares de la alfabetización en información, el público usuario reconoce la necesidad de información, analiza conociendo las opciones disponibles para cubrir esa necesidad, y piensa en cómo poder obtener la mejor información del recurso. El aporte establecido en este modelo radica en partir de los conocimientos que, gradualmente, adquiere el estudiantado, en la medida que crecen las exigencias del contexto y sus necesidades de información para el estudio, investigación y desempeño profesional, aptitudes estas que se incrementan con el tiempo. El pilar séptimo constituye la aptitud más alta que se debe alcanzar, ya que establece cómo acceder a la información y extraerla con el buen uso de las TIC. 
doi: http://dx.doi.org/10.15359/ree.22-2.15

URL: http://www.una.ac.cr/educare

CORREO: educare@una.cr

Inmediatamente, en Australia y Nueva Zelanda emplearon el modelo relacional fenomenológico, añadiendo a los principios de aprendizaje independiente y pensamiento, que se encuentra en la práctica constructivista, el desarrollo de valores personales que fomentan el uso crítico de la información, la adquisición de conocimientos sobre los entornos informativos y un estilo personal que facilita la interacción del estudiantado. Luego, en el año 2003, propusieron un "marco para la alfabetización informacional en Australia y Nueva Zelanda", basándose en principios generales de los que deducen que una persona alfabetizada se compromete a la construcción del nuevo conocimiento, al buen uso de la información, a la toma de decisiones, al aprendizaje en forma continua y a la solución de problemas con el buen uso de esa información (Quindemil, 2010).

En el marco de la alfabetización informacional, la utilización de herramientas virtuales de la web 2.0, como redes sociales, blogs, wikis, podcast, filtrado social, Twitter, ofrece grandes posibilidades que no podríamos imaginar hace tan solo algunos años (Lozano, 2010). Sin dudas, la innovación, la actitud y la filosofía 2.0, de las ideas, la innovación, la imaginación y el conocimiento compartido, deben ser una nueva línea estratégica en las aulas y en la biblioteca; en donde todos los actores puedan apostar por el uso de estas herramientas y percibirlas como una oportunidad y no como una amenaza, porque la socialización de los espacios contribuye a un mejor conocimiento y entendimiento de los sujetos participantes que utilizan estas herramientas de aprendizaje. En este sentido, la biblioteca tiene una asignatura pendiente y es la de vincular el hábito del público lector con la innovación, con propuestas diferentes, diversificadas y atractivas, tanto respecto estrategias de búsqueda como de lectura.

La realidad de las instituciones universitarias resulta compartida; estadísticas de cada casa de estudio, desarrollos teóricos y antecedentes de investigación dan cuenta de una problemática común. El alto índice de desgranamiento en el primer año de las carreras, así como el proceso ininterrumpido que este significa, donde las dificultades de comprensión y producción de textos académicos aparecen como obstáculos que se posicionan como causales directas (Alcia, Torres, Böhm, Terrera, Lucero, 2013; Vázquez, 2005), a los que las dificultades provenientes de la carente alfabetización informacional podrían sumarse.

\section{Resultados obtenidos del trabajo de campo}

\section{Diseño metodológico}

Como se ha dicho, en el marco de la estrategia metodológica cuantitativa propuesta, se escogió la técnica de encuesta, con un cuestionario como instrumento de recolección, elaborado por el equipo de investigación.

A partir de la revisión teórica y de antecedentes realizada, fueron seleccionadas aquellas variables más relevantes ajustadas a los cuestionamientos propuestos. Luego de operacionalizadas, 
se diseñó una alternativa rígida y estructurada de cuestionario, organizada en torno a los tres ejes de la investigación: fuentes de información, competencias de escritura y de lectura.

Para ello seretomaron dichos constructos teóricosy seestablecieron los aspectoso dimensiones a medir en cada uno de ellos, desde la perspectiva de los desarrollos teóricos asumidos. Este proceso técnico y reflexivo llevado a cabo el equipo de investigación tuvo por resultado un instrumento de medición compuesto por 20 ítems, mayoritariamente cerrados, de diversa modalidad de respuesta. Los sistemas de categorías de respuesta fueron construidos siguiendo los criterios de exhaustividad y exclusividad, en caso de corresponder, tal como la disciplina indica.

En dichas consignas, y como en estudios antecedentes (Vázquez, 2010), lo que se pretende medir mayoritariamente son hábitos de estudiantes, asumidos frente a las instancias que en el ámbito universitario se les presentan, respecto a leer, escribir o manejarse con información académica. Entendiendo, en este caso, un hábito como modo especial de proceder, no único sino como repetición de actos semejantes.

La intención es poder captar las acciones que efectivamente llevan a cabo cuando deber resolver este tipo de tareas, a sabiendas de que muchas veces existe distancia entre lo que dicen que hacen las personas estudiantes y lo que en realidad hacen (Vázquez, 2010).

La validez del instrumento confeccionado, entendida como la capacidad de brindar una imagen adecuada del concepto que se desea medir (Cea, 1999), se respalda en las definiciones teóricas que se han desarrollado y asumido en el marco teórico de esta investigación, y que han sido la base para la concreción de esas variables intermedias en múltiples indicadores, otorgando validez de contenido. Del mismo modo, la validez de constructo resulta de comparar los resultados obtenidos con las hipótesis de investigación, en la media que resultan convergentes $y$, por su intermedio, coherentes con la revisión de antecedentes que las inspiraron.

También es posible dotar el cuestionario aplicado de una cuota de fiabilidad (Cea, 1999), en tanto se obtuvieron resultados consistentes en las mediciones sucesivas que hicieron a lo largo del tiempo en las dos universidades en las que se concentró el estudio.

El trabajo de campo para su aplicación tuvo lugar a fines del ciclo lectivo 2012, cuando se interrogó estudiantado matriculado en la carrera de Profesorado de Grado Universitario en Educación General Básica, de la Facultad de Educación Elemental y Especial (FEEyE) de la UNCuyo; y a mitad de 2014 para las personas que cursaban en la Facultad de Psicología (FP) el inicio de las licenciaturas en Niñez, Adolescencia y Familia, Psicología y Criminalística de la UDA.

La selección de las 146 personas participantes respondió a una estrategia muestral no probabilística, que considerara las dos universidades estudiadas y las cuatro carreras en su interior escogidas. El estudiantado debía estar cursando la mitad del segundo año lectivo, de manera que pudiera dar cuenta de los aspectos indagados. 
doi: http://dx.doi.org/10.15359/ree.22-2.15

URL: http://www.una.ac.cr/educare

CORREO: educare@una.cr

En el caso de la FEEyE se realizaron 81 cuestionarios simples, autoadministrados. El margen de error considerado fue del $8 \%$ con una confianza del $92 \%$. El tamaño de la muestra recomendado para esa población y errores tolerados es de 80 casos, lo que se ajusta perfectamente al grupo muestral involucrado.

Para el caso de la FP se trabajó con una muestra compuesta por 65 formularios, también autoadministrados, distribuidos proporcionalmente entre las tres carreras, seleccionadas las unidades de modo aleatorio al interior de cada una, tolerando un error de $5 \%$ y un nivel de confianza del $95 \%$.

\section{Principales resultados y discusión}

De modo general, es oportuno afirmar que no se observaron diferencias sustanciales entre ambos momentos de la indagación, en cuanto al contexto institucional de educación universitaria pública, por un lado, y privada, por el otro, razón por la cual los resultados se expresan de manera conjunta y se hacen aclaraciones u observaciones en los casos que estas diferencias fueron sustanciales o significativas.

En relación con el primer eje, cuando se indagó sobre la biblioteca de la facultad, el 100\% del grupo de la FEEyE declara haber concurrido a la biblioteca alguna vez, mientras que el $62 \%$ de la FP dijo no haber ido nunca. Esto nos permite hipotetizar que la concurrencia se relaciona de modo directo con el sistema de acceso a los textos indicados como obligatorios para cada materia que cada institución ha cimentado. Para el grupo de la universidad privada el primer medio de acceso mencionado es la fotocopiadora y el segundo, internet.

Cuando se avanzó en la indagación, en relación con las estrategias que se utilizan para buscar información en el marco de la biblioteca (y solo considerando el grupo de la facultad pública), es interesante ver que frente a las categorías propuestas, la selección realizada se concentró con las mayores frecuencias en la pregunta al personal bibliotecario, el $67 \%$ de la muestra recurre a esta estrategia de búsqueda, seguida de un porcentaje menor, un 32,1\% que usa sus propios medios y la PC dispuesta para tal fin en la biblioteca.

También, se inquirió en relación con el número de veces que ha recurrido en busca de textos a la biblioteca y los resultados han sido los siguientes: en promedio el estudiantado ha recurrido 6 veces, sin embargo, ese resultado está influido por valores más dispersos, en tanto la mediana es 5 veces y el número modal de visitas es de 10. Para el grupo de la FP, el $23 \%$ ha concurrido entre 1 y 5 veces desde el inicio de su trayectoria universitaria.

En cuanto a la interrogación respecto de las posibilidades de acceso cotidiano a herramientas informáticas o tecnología para uso académico, el 94\% contestó de manera afirmativa. Luego se pidió que señalaran la más habitual de las herramientas y el 77\% declara 
utilizar PC de escritorio, lo que es seguido porcentualmente por el uso de notebook que se constituye en un $19 \%$ y celular en tercer lugar.

Este dato permite interrogarse en relación con las netbooks entregadas desde el año 2010 por el Programa Conectar Igualdad ${ }^{6}$ a estudiantes de nivel medio de educación pública. Es decir, el grupo de edades afín a la implementación de ese programa nacional no declara de manera mayoritaria contar con esta herramienta para uso habitual, si bien se desconoce si la escuela de origen es pública o privada. No es un tema menor, quedando tal vez planteada una línea de investigación futura que este equipo pudiera retomar y profundizar.

Así mismo, resulta interesante destacar que, si bien estamos en la era de la información de las comunicaciones y la informatización, aún hay registros desafiantes en relación con quiénes no cuentan con alguno de estos tipos de herramientas propuestos. De hecho, el 12,3\% del grupo declara no tener ninguna de las herramientas propuestas en propiedad.

Cuando se indagó acerca del conocimiento sobre las estrategias de búsqueda de información, fue elocuente la respuesta afirmativa por parte del 92\% del grupo de la FEEyE. Sin embargo, un 20 \% del grupo de la FP declaró no saber cómo buscar en internet el material académico, y esta razón, junto con la falta de indicaciones docentes y la carencia de dinero son mencionadas como los principales obstáculos en el acceso y relación con la información académica, como muestra el ranking presentado en la Tabla 1.

Tabla 1: Ranking de factores que dificultan el acceso o relación con la información académica

\begin{tabular}{lc}
\hline Factores & Orden \\
\hline No saber dónde buscarla & $1^{\circ}$ \\
Ausencia o ineficacia de las indicaciones de docentes & $2^{\circ}$ \\
Falta de dinero para adquirirla & $3^{\circ}$ \\
No disponer de tecnología & $4^{\circ}$ \\
Falta de compañeros o compañeras que aconsejen & $5^{\circ}$ \\
No tener familiares que orienten al respecto & $6^{\circ}$ \\
\hline
\end{tabular}

\footnotetext{
${ }^{6}$ El Programa Conectar Igualdad fue implementado desde el primer semestre del año 2010, a través de un decreto presidencial. Se propuso distribuir equipos netbooks a todo el alumnado y docentes de escuelas secundarias, de educación especial e institutos de formación docente de gestión estatal, los que estarían pensados tanto para uso en la escuela como en los hogares. Pretende, esta acción, recuperar y valorizar la escuela pública y reducir las brechas digitales, educativas y sociales en el territorio nacional, según menciona el sitio oficial http://www. conectarigualdad.gob.ar/seccion/sobre-programa/que-conectar-igualdad-53
} 
doi: http://dx.doi.org/10.15359/ree.22-2.15

URL: http://www.una.ac.cr/educare

CORREO: educare@una.cr

Hay que someter a reflexión esta situación, que promediando el cursado del segundo año de la vida universitaria declaran que no conocen cómo buscar información en la web, lo que remite nuevamente, como explicación, a la relación con el servicio de fotocopiado para el acceso a la bibliografía, y el hábito de pagar por ello, lo que no es de tal magnitud en la facultad pública.

La interrogación sobre el buscador más conocido tuvo en cada instrumento una suerte de combinación confusa, ya que algunas respuestas evidenciaron el poco conocimiento del estudiantado en relación con las estrategias de búsqueda y de buscadores propuestos por la red.

Sin embargo, hay que tener en cuenta algunos tópicos relevantes. La realidad indica que la mayoría de los buscadores existentes han quedado subsumidos, de alguna manera, a Google, han sido superados e incorporados por el buscador universal. Lo que se puso de manifiesto en las respuestas aportadas por el estudiantado es la confusión entre buscadores, navegadores y sitios concretos, como por ejemplo Wikipedia. Este también es una habilidad que debería reforzarse entre el estudiantado, atendiendo la utilidad que este conocimiento les puede proporcionar a lo largo de la vida universitaria.

Al profundizar estos aspectos, en la indagación realizada en relación con el primer criterio que se adopta para elegir entre los resultados obtenidos de una búsqueda, el 54\% declaró que su elección estaba condicionada por el hecho de que la información provenga de un sitio identificado como .org - .edu - .gov. Un 18,5\% utiliza un criterio más práctico y nada fundamentado desde los contenidos posibles de los resultados, en la medida que seleccionan el que aparece primero o segundo en la lista de resultados. Solo el $12 \%$ alude a la pertenencia de la información a circuitos científicos, razón que debería ser considerada a prima facie en el ámbito académico, sin embargo, ocupa el tercer lugar entre las prácticas concretas.

Han sido elocuentes los aportes teóricos en cuanto a la relevancia de la lectura y la escritura en el marco de la alfabetización académica, en razón de ello y también como forma de cumplimentar los objetivos propuestos en este estudio, se avanzó en el conocimiento empírico del tema. Para tal fin se examinó, acerca de varios aspectos en torno a rutinas de lectura y escritura circunscriptas específicamente al ámbito de los estudios universitarios.

En el marco de los aspectos centrales vinculados con la alfabetización académica, se puntualiza la indagación respecto a la lectura, a la escritura y a las dificultades comparativas que las mismas revisten. Al respecto, el $84 \%$ del grupo declara mayores dificultades a la hora de escribir un texto propio, frente al $16 \%$ que considera más dificultoso leer o comprender un texto nuevo.

De las competencias de escritura, se preguntó sobre las dificultades u obstáculos experimentados al desarrollar un texto requerido en el ámbito de la carrera de grado. En primer lugar, se mencionó el uso de un lenguaje académico o propio de la disciplina y luego se señaló la falta de hábito o ejercitación en escritura, como muestra la Tabla 2, donde las diferencias entre las tres primeras respuestas elegidas no fueron significativas. 
Tabla 2: Principales dificultades a la hora de realizar un texto de producción propia

1. Plasmar un lenguaje escrito académico, pertinente o propio de la disciplina de estudio.

2. No contar con hábitos o ejercitación previa de escritura.

3. Detectar desajustes entre las perspectivas del personal docente y el punto de vista propio.

4. Vincular o relacionar en el texto, las diversas teorías o conocimientos ya adquiridos.

Así mismo, un 79\% del estudiantado considera medianamente suficientes los conocimientos adquiridos hasta entonces, como para poder escribir correctamente un texto académico requerido.

En relación con la cantidad de trabajos escritos realizados, estimamos que la comprensión de la pregunta asumió diferentes andamiajes interpretativos en los grupos, desde la producción total de un escrito, hasta la realización de trabajos prácticos que implicaban escritura; esto estaría explicando la tan diversa cantidad declarada por el estudiantado. El 30\% del grupo declara haber realizado 20 trabajos escritos y el 15\% asume haber realizado 10.

Sobre quienes dijeron preferir los exámenes escritos frente a los orales, se avanzó un paso más y se indagó lo atinente a la modalidad de examen escrito que generaba mayor dificultad y los resultados fueron equivalentes entre múltiple opción y los que implican redacción y desarrollo de respuestas.

Finalmente, respecto las competencias de lectura, en primer lugar, se cuestionó acerca del número de veces que debían leer textos vinculados a la especificidad disciplinar para lograr su comprensión. Como es subjetiva y difícil de medir la apreciación que los sujetos indagados pueden tener al respecto, se les agregó un criterio que decía comprender, en el sentido de haber internalizado el texto de tal modo que fuera posible explicárselo a un compañero o compañera o dar cuenta de una síntesis de este mismo. El número que acumula la mayor frecuencia es 2 veces, reuniendo el $50 \%$ de las personas indagadas. Por otra parte, el $37 \%$ del grupo declaró que para lograr la comprensión propuesta la lectura debía realizarse 3 veces, lo que tiene otro tipo de implicancias, por ejemplo la inversión de una mayor carga de tiempo para lograr el objetivo propuesto. Finalmente, el 12\% expresó que la lectura debía realizarse entre 4 y 5 veces, lo que evidencia niveles interesantes de dificultad y graves carencias en sus competencias de lectura. Los datos se dispersan entre las menciones de 1 y 10 veces, donde 3 el promedio para el grupo indagado.

Las estrategias de lectura por primera vez de un texto académico refieren a identificar ideas principales, y luego a leer títulos, subtítulos e índices.

Por otro lado, al indagar sobre las herramientas más utilizadas para estudiar un texto luego de haber sido leído, el $89 \%$ de las personas menciona el resumen, seguida por el mapa conceptual (43\%) los cuestionarios (5\%), y luego por la memorización en un 35\%; ostentando este indicador ínfimas menciones para el resto de las alternativas que se presentaron a las personas indagadas, como se ve en la Figura 1. 
doi: http://dx.doi.org/10.15359/ree.22-2.15

URL: http://www.una.ac.cr/educare

CORREO: educare@una.cr

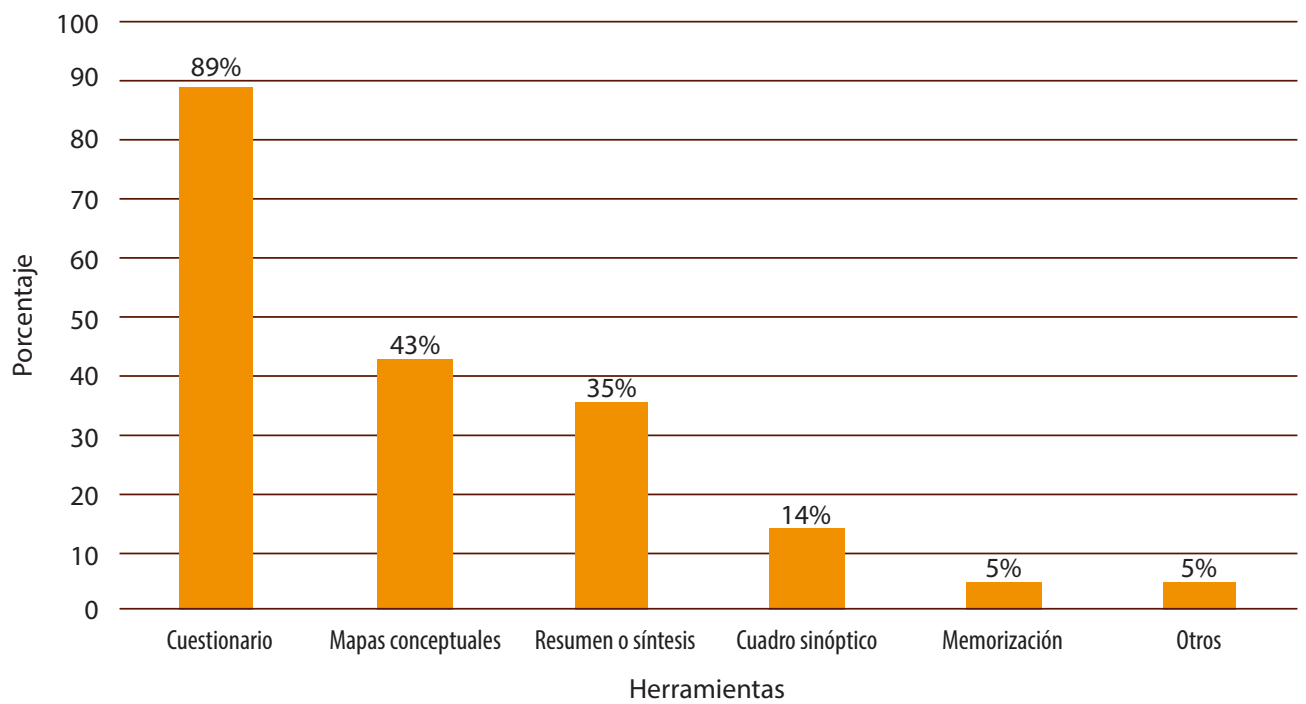

Figura 1: Herramientas más usadas al estudiar un texto ya leído. Opción múltiple

Este resultado denota la falta de creatividad, estrategias individuales y el escueto margen de acción que las personas estudiantes están habituadas a ejercer en un segundo año universitario, a la hora de internalizar los textos que le son indicados para estudiar.

Entre las principales dificultades que los textos académicos ostentan a quienes estudian, se encuentran la presencia en ellos de lenguaje no entendido y en segundo lugar la excesiva o gran extensión de estos. Es notable que la situación que menos se refiere de las sugeridas es aquella que menciona dificultades provenientes de material que se encuentra descontextualizado, sin referencia al texto del que provienen, siendo que ello es así toda vez que el sistema docenteservicio de fotocopiado es la estrategia más utilizada de búsqueda bibliográfica. Vale preguntarse si ello no es reflejo de una actitud naturalizadora que invisibiliza la carencia de estos marcos o referencias cada vez que quienes estudian deben situarse frente a un nuevo texto académico.

\section{Breves conclusiones y reflexiones}

Es oportuno recoger aquí los objetivos específicos del trabajo propuesto, y reflexionar en torno a su logro. Respecto a la intención inicial de indagar acerca de los conocimientos previos que se vinculan a las estrategias de búsqueda de información y relación con fuentes bibliográficas y manejo concreto de herramientas informáticas, resulta un aspecto pendiente, que por su propia complejidad y cualidad resultó postergado por el equipo de investigación para ser abordado en una instancia específica a futuro.

Sin duda se logró lo propuesto en torno a conocer la importancia de la denominada alfabetización universitaria y los alcances de esta desde perspectivas profesionales y teóricas. 
El tema se halla vinculado a cuantiosas aristas de interés para quienes se desempeñan en la actividad académica y la docencia universitaria, con lo cual resultó complejo el recorte y selección de aspectos y perspectivas; donde los desarrollos de Carlino (2009) y Barbosa-Chacón et al., (2010) son los principales aportes incorporados, por el alto grado de empatía geográfica y contextual de sus estudios y esta propuesta.

Ha sido posible profundizar sobre los aspectos favorecedores y obstaculizadores para el estudiantado universitario del manejo de la bibliografía, de las fuentes de información existentes y de estrategias de lectura y escritura, tal como en los objetivos se propuso el equipo.

En este sentido, todos los actores y las actoras que se desempeñan en el ámbito universitario pueden dar cuenta acerca de las dificultades que suele presentar el estudiantado en el proceso de enseñanza, pero poco se hace que supere la queja y la atribución reiterada de responsabilidades a instancias de formación previas.

Posiblemente, la premisa de formar en lo disciplinar no permite ver con claridad que es también tarea docente abocarse a formar en la alfabetización. Se debe profundizar la dedicación a la lectura y escritura del estudiantado para luego poder avanzar en impartir conocimientos disciplinares de manera exitosa. Tanto en cuanto a la compresión que puedan tener sobre estos mismos y las competencias que puedan ir generando, las que redundarán, sin lugar a dudas, en una apropiación de conocimiento más sólida y estable, lo que es, en definitiva, parte del objetivo de la formación.

La lectura y la escritura son habilidades consideradas básicas para transitar la vida universitaria y son, a la vez, las que más problemas contienen, por esto los cuerpos docentes no pueden ignorar el desafío que está presente en cada estudiante, en relación con poder superar o no las dificultades, lo que puede resultar, en el peor de los casos, en la deserción y cuanto menos en incursionar en un proceso de desgranamiento que tampoco garantiza un final feliz.

El desafío de trabajar en promover competencias en el estudiantado podría ser la llave que abra la puerta hacia el aprovechamiento de la riqueza que imparta la formación universitaria.

La indagación empírica ha dejado traslucir que el estudiantado cuenta con falencias en temas vinculados con la alfabetización académica; esto es, declaran tener dificultades en la lectura, en la comprensión de los textos y también en la escritura, y solo en ocasiones las instancias de evaluación evidencian estos problemas, lo que también nos deja reflexionando acerca de ellas.

Son varios los aspectos que nos someten a interrogarnos en cuanto a nuestra función y a los pasos previos necesarios que deberíamos, en nuestra calidad de docentes, dar con el estudiantado para forjar en él herramientas útiles para la vida universitaria. El hecho de que el estudiantado no sepa buscar adecuadamente información académica nos interroga en relación con carencias, en cuanto a la necesidad y oportunidad de proporcionarles conocimientos en esos sentidos, transversalmente a la dinámica disciplinar. 
doi: http://dx.doi.org/10.15359/ree.22-2.15

URL: http://www.una.ac.cr/educare

CORREO: educare@una.cr

Otra cuestión podría vincularse a deficiencias en la amigabilidad con los textos y el acceso a material mediado, lo que hace que, en la mayoría de las ocasiones, el estudiantado pueda obviar la consulta directa del texto, reemplazado por fotocopias o resúmenes ajenos. Esto da por resultado un sujeto lector descontextualizado respecto del material teórico, que solo accede a trozos de estos, que desconoce el producto como unidad textual y resultante de un determinado autor, tiempo y espacio.

En el mismo sentido es digno de mencionarse el escaso criterio y el desconocimiento sobre lo que realmente pesa a la hora de seleccionar un artículo dentro de un cúmulo de resultados obtenidos en relación con un tema. Consideramos que esos criterios hacen el nuevo rol docente y bibliotecario, y deben ser impartidos en los primeros pasos en la universidad, ya que acompañarán al estudiantado, cuando menos, por el resto del recorrido.

En síntesis, lo que pone sobre la mesa y al desnudo este trabajo es que urge la transferencia y formación en competencias académicas, que los datos resultantes de las indagaciones claman por este tipo de empeño, y que las mismas competencias se traducirán, sin duda, en beneficios para la vida académica, tanto de quienes recién comienzan, como de las personas docentes y bibliotecarias que les acompañan.

Queda pendiente, a partir de este desarrollo, profundizar numerosos aspectos vinculados a la problemática y que, por el alcance de la propuesta, no pueden ser incluidos en este estudio, pero que, sin lugar a dudas y tal como se anticipó, serán temas de próximas investigaciones.

\section{Referencias}

Alcia, T., Torres, L. M., Böhm, F. M., Terrera, S. E. y Lucero, A. E. (2013). La elaboración de la tesina como la última cuesta en la graduación: Sus dificultades y el rol de tutoría. Revista Electrónica Educare, 17(2), 123-144. Recuperado de http://www.revistas.una.ac.cr/index. php/EDUCARE/article/view/5024/4807

Barbosa-Chacón, J. W., Barbosa-Herrera, J. C., Marciales-Vivas, G. P. y Castañeda-Peña, H. A. (2010). Reconceptualización sobre competencias informacionales. Una experiencia en la educación superior. Revista de Estudios Sociales, 37, 121-142. Recuperado de http://www. redalyc.org/articulo.oa?id=81519011007

Caldera, R. y Bermúdez, A. (2007). Alfabetización académica: Comprensión y producción de textos. Educere, 11(37), 247-255. Recuperado de http://www.redalyc.org/articulo. oa? id $=35603710$

Carlino, P. (2009). Escribir, leer y aprender en la Universidad: Una introducción a la alfabetización académica. Buenos Aires: UBA. Recuperado de http://www.relacionesdeltrabajo.fsoc.uba. ar/prod/alfabetizacion\%20academica.pdf 
Carlino, P. (2013). Alfabetización académica diez años después. Revista Mexicana de Investigación Educativa, 18(57), 355-381. Recuperado de http://www.redalyc.org/pdf/140/14025774003.pdf

Cea, M. (1999). Metodología cuantitativa: Estrategias y técnicas de investigación social. Madrid: Síntesis.

Goyes, A. y Klein, I (2012). Alcances, limitaciones y retos en la enseñanza de la escritura en la universidad (dos casos: Colombia y Argentina). En L. Laco, L. Natale y M. Ávila (Comps.), La lectura y la escritura en la formación académica, docente y profesional (pp. 6-14). Buenos Aires, Argentina: Editorial UTN. Recuperado de http://www.edutecne.utn.edu.ar/lectura escritura/lectura escritura.pdf

Lozano, R. (2010). Fomento de la lectura en la biblioteca pública 2.0: Una apuesta por la innovación y el riesgo. Anuario ThinkEPI, 4, 87-90. Recuperado de https://dialnet.unirioja. es/servlet/articulo?codigo $=3263856$

Marciales-Vivas, G. P., González-Niño, L., Castañeda-Pena, H. y Barbosa-Chacón, J. W. (2008). Competencias informacionales en estudiantes universitarios: Una reconceptualización. Universitas Psychologica, 7(3), 643-654. Recuperado de http://www.scielo.org.co/pdf/rups/ v7n3/v7n3a04.pdf

Quindemil, E. (2010). Introducción de la alfabetización informacional en la especialidad de bibliotecología y ciencias de la información. Acimed, 21(1), 99-110. Recuperado de http:// scielo.sld.cu/pdf/aci/v21n1/aci08110.pdf

Torino, M. E. (2012) La alfabetización académica en alumnos pertenecientes a comunidades originarias. En L. Laco, L. Natale y M. Ávila (Comps.), La lectura y la escritura en la formación académica, docente y profesional (pp. 325-329). Buenos Aires, Argentina:Editorial UTN. Recuperado de http://www.edutecne.utn.edu.ar/lectura escritura/lectura escritura.pdf

Varela, C. (2009). Adquisición de competencias en información mediante e-Learning: Una responsabilidad compartida entre docencia y biblioteca. Ibersid, 3, 303-312. Recuperado de http://www.ibersid.eu/ojs/index.php/ibersid/article/view/3757/3518

Vázquez, A. (2005). ¿Alfabetización en la Universidad? Colección de cuadernillos de actualización para pensar la enseñanza universitaria, 1(1), 1-12, Recuperado de http://www.eemn1tsas. edu.ar/Autoevaluacion/Vasquez-Alfabetizacion\%20en\%20la\%20Universidad.pdf

Vázquez, A. (2010). ¿Qué dicen que hacen los estudiantes universitarios cuando escriben desde fuentes? En A.Vázquez, M. Novo, I. Jakob y L. Pelliza (Comps.), Lectura, escritura y aprendizaje disciplinar (pp. 153-174). Córdoba, Argentina: UniRío editora. Recuperado de https://www. unrc.edu.ar/unrc/digtal/libro jornadas unesco unrc 2010.pdf 